\title{
Imaging goes label-free
}

Researchers develop a high-sensitivity, label-free imaging technology based on stimulated Raman scattering.

Fluorescence imaging, although undoubtedly powerful for cell biology, usually requires labeling the molecular species of interest in some way. This involves adding bulky appendages to the molecule, which can interfere with normal function, and the fluorophores themselves are subject to photobleaching, limiting imaging time.

Raman spectroscopy, which can be used to identify molecules based on signature vibrational frequencies of chemical bonds following excitation by a laser, offers a labelfree alternative. Conventional Raman, however, is too insensitive to be very useful for subcellular imaging. Seeking a more sensitive alternative, Sunney Xie, of Harvard University, and his colleagues developed the technology of coherent anti-Stokes Raman scattering (CARS) microscopy 10 years ago. CARS is a nonlinear Raman technique in which two lasers (a pump beam and a Stokes beam) interact with the sample, causing identical molecules to vibrate in phase, generating an enhanced anti-Stokes signal. CARS is thus much more sensitive than conventional Raman microscopy. However, the resulting CARS spectrum contains a strong background, which makes assigning all but well-isolated bond frequencies very difficult.

Xie, along with co-first authors Christian Freudiger and Wei Min, and their colleagues, now describe a new Raman-based imaging technique that overcomes the problem of spectral background in CARS microscopy and is even more sensitive than CARS. This new technology, which is based on stimulated Raman scattering (SRS), also trains a pump beam and a Stokes beam on the sample; when the difference in laser frequencies matches a particular molecular frequency, the Raman signal is amplified. When the difference frequency does not match any molecular frequency, no signal is generated, which eliminates the spectral background. Thus the SRS spectrum is essentially identical to a conventional Raman spectrum, making spectral assignment much easier than in CARS.

With this development of SRS microscopy, "CARS is almost obsolete," says Xie. SRS microscopy allows three-dimensional imaging of specific molecular species to a depth

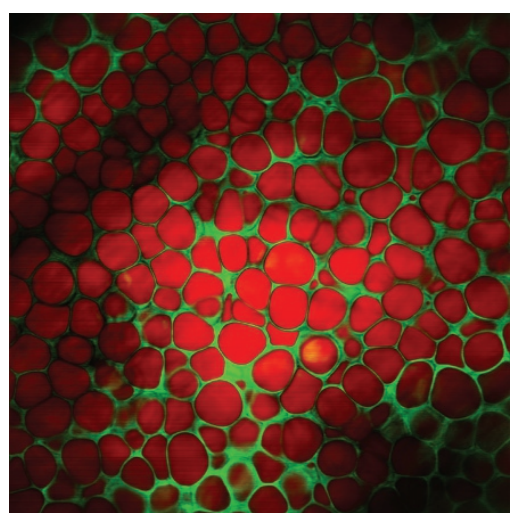

Two-color SRS microscopy image of DMSO (green) and lipid (red) in the subcutaneous fat layer in mouse skin. Image courtesy of the Xie lab.

of about 0.3 millimeters. For in vivo imaging applications, the researchers are building endoscopes. "The potential for medical imaging is enormous," says Xie. Like CARS, video-rate imaging should be possible with SRS, though the researchers have not yet tested this.

SRS microscopy should be particularly useful for imaging lipids and small molecules such as drugs. Working with Jing Kang of Harvard Medical School, Xie and his colleagues imaged omega- 3 fatty acid uptake in human cells. "Lipids are an uncharted area in biology;...imaging them in situ, in real time, would allow the study of lipid metabolism," notes Xie. Working with Jason Tsai of Pfizer, the researchers also monitored drug delivery into mouse skin, specifically dimethyl sulfoxide (DMSO), a skin-penetrating enhancer, and retinoic acid, a common acne treatment.

Switching over to SRS microscopy should be relatively straightforward for researchers who already have CARS microscopes set up, with a few small modifications. Commercial CARS microscopes are also expected to become available soon. "Until there is a good commercial product, the applications will always be limited to a handful of specialists," notes Xie. "So that's why we've been working hard with microscope companies to try to make them available to everyone."

\section{Allison Doerr}

\section{RESEARCH PAPERS}

Freudiger, C.W. et al. Label-free biomedical imaging with high sensitivity by stimulated Raman scattering microscopy. Science 322, 1857-1861 (2008). 